\title{
ON THE EXISTENCE OF TURYN SEQUENCES OF LENGTH LESS THAN 43
}

\author{
GENET M. EDMONDSON, JENNIFER SEBERRY, AND MALCOLM R. ANDERSON
}

\begin{abstract}
Some theoretical results and computational algorithms are described which verify previous calculations, prove some theoretical nonexistence results, and totally enumerate all inequivalent Turyn sequences of length less than 43, that is, the longer sequence has length less than 43 .
\end{abstract}

\section{INTRODUCTION}

Engineers concerned with finding the exact distance from their Earth base to the Moon, Venus, and the other planets, or even to a moving aircraft, use radar signals which consist of sequences of binary entries, effectively 1 and -1 . Unfortunately, they have found that the type of single sequences they prefer to use, known as Barker sequences, apparently do not exist for lengths greater than 13.

A desire for longer usable sequences has prompted research engineers to consider sets of two or more binary sequences for these problems and others concerned with range, depth, or information compression.

The use of similar sequences in searching for the existence of Hadamard matrices and weighing matrices ultimately led to the development of Turyn sequences. However, before we explain these, we need some definitions of the terms we will use.

Definition 1 (Nonperiodic autocorrelation function). Let $X=\left\{\left\{a_{11}, \ldots, a_{1 n}\right\}\right.$, $\left.\left\{a_{21}, \ldots, a_{2 n}\right\}, \ldots,\left\{a_{m 1}, \ldots, a_{m n}\right\}\right\}$ be $m$ sequences of elements 1 and -1 or 0,1 , and -1 of length $n$. In many cases we will have a short sequence which we will extend to length $n$ by adding zeros to the end.

The nonperiodic autocorrelation function of the family of sequences $X$, denoted by $N_{X}$, is a function defined by

$$
N_{X}(j)=\sum_{i=1}^{n-j}\left(a_{1, i} a_{1, i+j}+a_{2, i} a_{2, i+j}+\cdots+a_{m, i} a_{m, i+j}\right),
$$

Received by the editor June 26, 1991 and, in revised form, January 20, 1992 and September 25, 1992.

1991 Mathematics Subject Classification. Primary 94A11, 68R05.

The research of the second author was funded by Telecom grant 7027, ARC grant A49130102, and an ATERB grant and was written while on faculty of the Department of Electrical Engineering and the Department of Computer Science and Engineering, University of Nebraska, Lincoln, NE 68588 , USA. 
where $j$ can range from 1 to $n-1$. For a single sequence $X=\left\{x_{1}, \ldots, x_{n}\right\}$ this can be written as

$$
N_{X}(j)=\sum_{i=1}^{n-j} x_{i} x_{i+j}
$$

We can also think of this in terms of matrices. If we form the following collection of $m$ matrices of order $n$,

$$
\left[\begin{array}{cccc}
a_{11} & a_{12} & \cdots & a_{1 n} \\
0 & a_{11} & & a_{1, n-1} \\
\vdots & & \ddots & \\
0 & \cdots & 0 & a_{11}
\end{array}\right], \ldots,\left[\begin{array}{cccc}
a_{m 1} & a_{m 2} & \cdots & a_{m n} \\
0 & a_{m 1} & & a_{m, n-1} \\
\vdots & & \ddots & \\
0 & \cdots & 0 & a_{m 1}
\end{array}\right]
$$

then $N_{X}(j)$ is simply the sum of the inner products of rows 1 and $j+1$ of these matrices.

Definition 2 (Periodic autocorrelation function). The periodic autocorrelation function of the family of sequences $X$, denoted by $P_{X}$, is a function defined by

$$
P_{X}(j)=\sum_{i=1}^{n}\left(a_{1, i} a_{1, i+j}+a_{2, i} a_{2, i+j}+\cdots+a_{m, i} a_{m, i+j}\right),
$$

where we assume the second subscript is actually chosen from the complete set of residues modulo $n$, that is, $i+j$ is really $(i+j) \bmod n$. As before, $j$ can range from 1 to $n-1$.

Again we can interpret the function $P_{X}$ in matrix terms. If we form the $m$ circulant matrices which have the $m$ sequences of $X$ as their first rows, that is,

$$
\left[\begin{array}{cccc}
a_{11} & a_{12} & \cdots & a_{1 n} \\
a_{1 n} & a_{11} & \cdots & a_{1, n-1} \\
& & \ddots & \\
a_{12} & a_{13} & \cdots & a_{11}
\end{array}\right], \ldots,\left[\begin{array}{cccc}
a_{m 1} & a_{m 2} & \cdots & a_{m n} \\
a_{m m} & a_{m 1} & \cdots & a_{m, n-1} \\
& & \ddots & \\
a_{m 2} & a_{m 3} & \cdots & a_{m 1}
\end{array}\right],
$$

then $P_{X}(j)$ is the sum of the inner products of rows 1 and $j+1$ of these matrices.

Definition 3 (Complementary sequences). If $X$ is a family of sequences of length $n$ as above with a zero nonperiodic autocorrelation function, that is, $N_{X}(j)=0$ for $j=1,2, \ldots, n-1$, then we will call $X$ the class of $m$ complementary sequences of length $n$.

The following results follow from these definitions.

Proposition 4. Let $X$ be a family of sequences as above. Then

$$
P_{X}(j)=N_{X}(j)+N_{X}(n-j), \quad j=1, \ldots, n-1 .
$$

Note that $P_{X}(j)$ may equal 0 for all $j=1, \ldots, n-1$, even though the $N_{X}(j)$ do not.

Corollary 5. If $N_{X}(j)=0$ for all $j=1, \ldots, n-1$, then $P_{X}(j)=0$ for all $j=1, \ldots, n-1$.

\section{Golay SEQuences}

Definition 6 (Golay sequences and Barker sequences). If $X$ consists of two sequences $X=\left\{\left\{a_{1}, \ldots, a_{n}\right\},\left\{b_{1}, \ldots, b_{n}\right\}\right\}$, where $a_{i}, b_{j} \in\{1,-1\}$ and 
$N_{X}(j)=0$ for $j=1, \ldots, n-1$, then the sequences in $X$ are called Golay complementary sequences of length $n$. If $X$ consists of a single sequence $X=$ $\left\{a_{1}, \ldots, a_{n}\right\}$, with $a_{i} \in\{0,1\}$ and $N_{X}(j)=1$ for $j=1, \ldots$, then we call $X$ a Barker sequence.

Barker sequences were studied first, but the longest length that could be found (and it is conjectured will be found) is 13 . So the search turned to binary sequences, particularly Golay sequences.

Golay sequences arose from Golay's early work $[6,7]$ on pairs of sequences with elements 1 and -1 and zero nonperiodic autocorrelation function. However Welti [20], Tseng [16], and Tseng and Liu [17] approached the subject from the point of view of two orthonormal vectors, each corresponding to one of two orthogonal waveforms. Later work, including Turyn's [18, 19], used four or more sequences and led to the development of what we call Turyn sequences.

Example. The following are Golay sequences where - means -1 .

\begin{tabular}{|c|c|}
\hline$n$ & Golay sequences \\
\hline 2 & $\begin{array}{ll}1 & 1 \\
1 & -\end{array}$ \\
\hline 10 & $\begin{array}{l}1--1-1---1 \\
1-----11-\end{array}$ \\
\hline 26 & $\begin{array}{c}111--111-1-----1-11--1---- \\
---11---1-11-1-1-11--1-\ldots\end{array}$ \\
\hline
\end{tabular}

There has been an extensive search for Golay sequences, but except for $\mathrm{Tu}$ ryn's result that these sequences exist for all lengths of the form $2^{a} 10^{b} 26^{c}$, where $a, b$, and $c$ are nonnegative integers, all other results have proved negative.

Geramita and Seberry [5, pp. 133-137], Andres [1], and James [9] have studied the smaller values of $n$ in Turyn's lemma, showing that the only Golay sequences of order $\leq 68$ which exist have orders $2,4,8,10,16,20,26,32$, 40,52 , and 64. Malcolm Griffin [8] has shown no Golay sequences can exist for lengths $n=2 \cdot 9^{t}$. The value $n=18$ was initially excluded by a complete search and is now theoretically excluded by Griffin's theorem and independently by Kruskal [14] and C. H. Yang [22, 23].

Recent theoretical work by Koukouvinos, Kounias, and Sotirakoglou [11] and Eliahou, Kervaire, and Saffari [4] shows that Golay sequences do not exist for $n=2 p$,where $p$ has any prime factor $\equiv 3(\bmod 4)$. This means the unresolved cases $<200$ are $N=74,82,106,116,122,130,136,146,148,164,170$, 178,194 .

For the study of the existence of Hadamard matrices and weighing matrices [5] the search for sequences has turned to searching for four (or more) sequences with elements $\{1,-1\}$ or $\{0,1,-1\}$, or of commuting variables or orthonormal elements with zero autocorrelation. 
One initial approach, suggested by Turyn in [18], has been studied carefully as it appears powerful and hopeful, giving rise to what are known as Turyn sequences. Alas, except for nine cases we give in detail in this paper, the largest being $n=14$, present evidence points to a similar outcome for Turyn sequences to that for Barker and Golay sequences: that they do not exist for longer lengths.

\section{TURYN SEQUENCES}

Definition 7 (Turyn sequences). Turyn sequences of length $t=n+1$ are four $(1,-1)$ sequences $X=(A, B, C, D)$ of lengths $n+1, n+1, n, n$ which have $N_{X}(j)=0$ for all $j=1, \ldots, n$ and in addition satisfy certain symmetry conditions, namely

(i) if $n$ is odd they have the following structure

$$
\begin{aligned}
A & =\left\{1,1, a_{2}, \ldots, a_{m},-a_{m}, \ldots,-a_{2},-1,-1\right\}, \\
B & =\left\{1,1, b_{2}, \ldots, b_{m},-b_{m}, \ldots,-b_{2},-1,1\right\}, \\
C & =\left\{1, c_{1}, c_{2}, \ldots, c_{m-1}, c_{m}, c_{m-1}, \ldots, c_{2}, c_{1}, 1\right\}, \\
D & =\left\{1, d_{1}, d_{2}, \ldots, d_{m-1}, d_{m}, d_{m-1}, \ldots, d_{2}, d_{1}, 1\right\},
\end{aligned}
$$

where $n=2 m+1$, or

(ii) if $n$ is even they have the following structure

$$
\begin{aligned}
A & =\left\{1,1, a_{2}, \ldots, a_{m-1}, a_{m}, a_{m-1}, \ldots, a_{2}, 1,1\right\}, \\
B & =\left\{1,1, b_{2}, \ldots, b_{m-1}, b_{m}, b_{m-1}, \ldots, b_{2}, 1,-1\right\}, \\
C & =\left\{1, c_{1}, \ldots, c_{m-1},-c_{m-1}, \ldots,-c_{1},-1\right\}, \\
D & =\left\{1, d_{1}, \ldots, d_{m-1},-d_{m-1}, \ldots,-d_{1},-1\right\},
\end{aligned}
$$

where $n=2 m$.

For more details see [12, 18] and [5]. In particular, we see in [5] that $N_{X}(j)$ being zero and the elements of the sequences being limited to \pm 1 implies that for odd $n$ we can express $4 n-2$ as the sum of two squares. Similarly, for even $n$ it must be possible to express $4 n+2$ as the sum of two squares.

The existence of a decomposition for $4 n \pm 2$ as the sum of two squares gives us a way to rule out the existence of Turyn sequences for some sizes. Specifically, if there is no decomposition for $4 n-2$ when $n$ is odd, there are no Turyn sequences of length $t=n+1$. The same thing happens for $4 n+2$ when $n$ is even. We also have the following result.

Lemma 8. If Turyn sequences fail the sum of squares condition for the odd length $t=n+1$, they do not exist for the next even length $t=n+2$.

Proof. If $4 n+2 \neq$ sum of the two squares for sequences of length $t=n+1$, where $n$ is even, then the condition for there to be sequences of length $t=n+2$ is that $4(n+1)-2=4 n+2$ must be the sum of two squares. This is clearly not satisfied.

We can use the following theorem from elementary number theory (see, for example, H. Davenport [2]) to help check whether such decompositions exist.

Theorem 9. Let $m=2^{e_{2}} 3^{e_{3}} 5^{e_{5}} \cdots p_{i}^{e_{p i}}$ be the prime factorization of an integer $m$. Then $m$ is a sum of two squares if and only if $e_{p_{i}}$ is even for all $p_{i} \equiv 3(\bmod 4)$.

Example. $42=2 \cdot 3 \cdot 7$ is not the sum of two squares, but $18=2 \times 3^{2}=$ $3^{2}+3^{2}$ is. 
This means we know immediately that there are no Turyn sequences for the following small lengths.

\begin{tabular}{l}
\hline \multicolumn{6}{|c|}{ no Turyn sequences } \\
\begin{tabular}{|c|c|c|c|c|c|}
\hline$n$ & 10 & 16 & 28 & 34 & 38 \\
\hline$t$ & 11 & 17 & 29 & 35 & 39 \\
\hline $4 n+2$ & 42 & 66 & 114 & 138 & 154 \\
\hline$n$ & 11 & 17 & 29 & 35 & 39 \\
\hline$t$ & 12 & 18 & 30 & 36 & 40 \\
\hline $4 n-2$ & 42 & 66 & 114 & 138 & 154 \\
\hline
\end{tabular}
\end{tabular}

There are a number of constructions that we can use to simplify the form of the Turyn sequences we seek. Turyn originally gave a proof that $a_{1}=1$ in [18], and we rely on his discussion of equivalence, which is used to shorten the computer search time and space and to ensure that we have found all sequences up to equivalence.

3.1. General form of the equations. The equations arising from the zero nonperiodic autocorrelation function can be written treating the elements of the four Turyn sequences as variables to be found. The full forms of these equations are not included here as they are too long. The details are given in [3]. We end up with $n-2$ equations in about $2 n$ variables. Including the constraint that each element is \pm 1 adds roughly a further $2 n$ equations.

The following example for the case $n=12$ illustrates what these equations are like.

Example. Equations for $n=12$ :

$$
\begin{array}{r}
a_{2}-c_{1}-d_{1}+1=0, \\
a_{3}+a_{2}+b_{2}-c_{2}-d_{2}-1=0, \\
a_{4}+a_{3}+b_{3}-c_{3}-d_{3}+1-c_{1} c_{2}-d_{1} d_{2}=0, \\
a_{5}+a_{4}+b_{4}-c_{4}-d_{4}-1+a_{2} a_{3}+b_{2} b_{3}-c_{1} c_{3}-d_{1} d_{3}=0, \\
a_{6}+a_{5}+b_{5}-c_{5}-d_{5}+1 \\
+a_{2} a_{4}+b_{2} b_{4}-c_{1} c_{4}-c_{2} c_{3}-d_{1} d_{4}-d_{2} d_{3}=0, \\
a_{5}+a_{6}+b_{6}+c_{5}+d_{5}-1 \\
+a_{2} a_{5}+a_{3} a_{4}+b_{2} b_{5}+b_{3} b_{4}-c_{1} c_{5}-c_{2} c_{4}-d_{1} d_{5}-d_{2} d_{4}=0, \\
a_{4}+a_{5}+b_{5}+c_{4}+d_{4}+1 \\
+a_{2} a_{6}+a_{3} a_{5}+b_{2} b_{6}+b_{3} b_{5} \\
+c_{1} c_{5}-c_{2} c_{5}-c_{3} c_{4}+d_{1} d_{5}-d_{2} d_{5}-d_{3} d_{4}=0, \\
a_{3}+a_{4}+b_{4}+c_{3}+d_{3}-1 \\
+a_{2} a_{5}+a_{3} a_{6}+a_{4} a_{5}+b_{2} b_{5}+b_{3} b_{6}+b_{4} b_{5} \\
+c_{1} c_{4}+c_{2} c_{5}-c_{3} c_{5}+d_{1} d_{4}+d_{2} d_{5}-d_{3} d_{5}=0,
\end{array}
$$




$$
\begin{gathered}
a_{2}+a_{3}+b_{3}+c_{2}+d_{2}+1 \\
+a_{2} a_{4}+a_{3} a_{5}+a_{4} a_{6}+b_{2} b_{4}+b_{3} b_{5}+b_{4} b_{6} \\
+c_{1} c_{3}+c_{2} c_{4}+c_{3} c_{5}-c_{4} c_{5}+d_{1} d_{3}+d_{2} d_{4}+d_{3} d_{5}-d_{4} d_{5}=0 \\
a_{1}+a_{2}+b_{2}+c_{1}+d_{1}-1 \\
+a_{2} a_{3}+a_{3} a_{4}+a_{4} a_{5}+a_{5} a_{6}+b_{2} b_{3}+b_{3} b_{4}+b_{4} b_{5}+b_{5} b_{6} \\
+c_{1} c_{2}+c_{2} c_{3}+c_{3} c_{4}+c_{4} c_{5}+d_{1} d_{2}+d_{2} d_{3}+d_{3} d_{4}+d_{4} d_{5}=0
\end{gathered}
$$

We can simplify these general equations using modulo 4 arithmetic and the following lemma.

Lemma 10. If $a, b= \pm 1$, then $a+b \equiv a b+1 \bmod 4$.

Again, the modulo 4 forms of the equations from the zero nonperiodic autocorrelation function are too long to give here. The details may be found in [3]. Using the modulo 4 form of the equations, we can prove the following result.

Lemma 11. For Turyn sequences with the shorter sequences of length $n=1+3 s$, where $s=1,2, \ldots$, there holds

$$
b_{s}=b_{s+1} \text {, }
$$

and where the length of the shorter sequence is $n=2+3 s$, where $s=1,2, \ldots$, there holds

$$
a_{s}=a_{s+1} \text {. }
$$

Proof. These results come from adding or subtracting the modulo 4 equations $j=s$ and $j=s+1$ for the relevant $n$, and remembering that $2(x+y) \equiv$ $0 \bmod 4$ if $x, y= \pm 1$. We label the equations so that equation $j$ comes from $N_{X}(j)=0$.

Using a combination of the long form of the equations and the modulo 4 equations, we can prove the following theorem.

Theorem 12. There are no Turyn sequences of length $n=8$, that is, where the longest sequences has length $t=n+1=9$.

Proof. We start with the six mod 4 equations and write them as a matrix:

$$
\left[\begin{array}{rrrrrrrrrrrr}
1 & 0 & 0 & 0 & 0 & 0 & -1 & 0 & 0 & -1 & 0 & 0 \\
1 & 1 & 0 & 1 & 0 & 0 & 0 & -1 & 0 & 0 & -1 & 0 \\
0 & 1 & 1 & 0 & 1 & 0 & -1 & -1 & -1 & -1 & -1 & -1 \\
1 & 0 & 1 & 1 & 1 & 1 & -1 & 0 & 0 & -1 & 0 & 0 \\
0 & 1 & 1 & 1 & 1 & 1 & 1 & 0 & 0 & 1 & 0 & 0 \\
0 & 0 & 1 & 0 & 0 & 1 & 0 & 0 & 1 & 0 & 0 & 1
\end{array}\right] \quad\left[\begin{array}{l}
a_{4} \\
b_{2} \\
b_{3} \\
b_{4} \\
c_{1} \\
c_{2} \\
c_{3} \\
d_{1} \\
d_{2} \\
d_{3}
\end{array}\right]=\left[\begin{array}{r}
-1 \\
1 \\
1 \\
-1 \\
-1 \\
0
\end{array}\right]
$$


Row reduction eventually gives

$\left(E 2^{\prime}\right)$

$$
\left[\begin{array}{rrrrrrrrrrrr}
1 & 0 & 0 & 0 & 0 & 0 & -1 & 0 & 0 & -1 & 0 & 0 \\
0 & 1 & 1 & 1 & 1 & 1 & 1 & 0 & 0 & 1 & 0 & 0 \\
0 & 0 & 1 & 1 & 1 & 1 & 0 & 0 & 0 & 0 & 0 & 0 \\
0 & 0 & 0 & 1 & 1 & 0 & 0 & 0 & -1 & 0 & 0 & -1 \\
0 & 0 & 0 & 0 & 1 & 0 & 0 & 1 & -1 & 0 & 1 & -1 \\
0 & 0 & 0 & 0 & 0 & 1 & 2 & 2 & 1 & 2 & 2 & 1
\end{array}\right]\left[\begin{array}{l}
a_{2} \\
a_{3} \\
a_{4} \\
b_{2} \\
b_{3} \\
b_{4} \\
c_{1} \\
c_{2} \\
c_{3} \\
d_{1} \\
d_{2} \\
d_{3}
\end{array}\right]=\left[\begin{array}{r}
-1 \\
-1 \\
0 \\
0 \\
1 \\
-1
\end{array}\right]
$$

From equations $\left(E 1^{\prime}\right),\left(E 3^{\prime}\right)$, and $\left(E 4^{\prime}\right)$ we find that $a_{4}=1$. We also know from the previous lemma that $a_{2}=a_{3}$ as $n=8=2+3 s$, where $s=2$. The remainder of these calculations work with integers rather than modulo 4 arithmetic.

We substitute these results into the general equations for $n=8$ to give us six new equations:

$\left(E 6^{\prime \prime}\right)$

$\left(E 5^{\prime \prime}\right)$

$\left(E 4^{\prime \prime}\right)$

$\left(E 3^{\prime \prime}\right)$

$\left(E 2^{\prime \prime}\right)$

$\left(E 1^{\prime \prime}\right)$

$$
\begin{array}{r}
a_{2}-\left(c_{1}+d_{1}\right)+1=0 \\
2 a_{2}+b_{2}-\left(c_{2}+d_{2}\right)-1=0 \\
a_{2}+b_{3}-\left(c_{3}+d_{3}\right)-c_{1} c_{2}-d_{1} d_{2}+2=0 \\
a_{2}+b_{4}+\left(c_{3}+d_{3}\right)+b_{2} b_{3}-c_{1} c_{3}-d_{1} d_{3}+1=0 \\
3 a_{2}+b_{3}+b_{2} b_{4}+\left(c_{2}+d_{2}\right)+c_{3}\left(c_{1}-c_{2}\right)+d_{3}\left(d_{1}-d_{2}\right)+1=0 \\
2 a_{2}+b_{2}+b_{2} b_{3}+b_{3} b_{4}+\left(c_{1}+d_{1}\right)+c_{2}\left(c_{1}+c_{3}\right)+d_{2}\left(d_{1}+d_{3}\right)+1=0
\end{array}
$$

These can be combined to give

$$
2 b_{2}+2 b_{3}+b_{4}+2 b_{2} b_{3}+b_{2} b_{4}+b_{3} b_{4}=-10 a_{2}-5 \text {. }
$$

Given that all the $b_{i}$ must be \pm 1 , the left-hand side cannot evaluate to -15 , so we must have $a_{2}=-1$. Using the fact that the right-hand side must be 5 and adding $2-2 b_{2} b_{3}$ to both sides shows that we must have $b_{2}=-b_{3}$. The equation then reduces to $b_{4}=7$, which is neither 1 nor -1 and hence gives us a contradiction.

3.2. Searching for Turyn sequences with a computer. The most basic way to search for Turyn sequences with a computer is to use a brute force approach, treating each sequence as a binary number, and to simply try all possible sequences until a satisfactory group is found. However, the amount of time increases exponentially with each increase in the length of the sequences being searched for. Around $n=18$ this starts to take about a CPU day on a DEC10.

To cut down on this increase, we tried writing the equations from the nonperiodic autocorrelation function explicitly. We then wrote a program to find solutions to these equations. 
Initially, we programmed the equations for each $n$ individually, but it became obvious that this was both too tedious and prone to error. Instead, a program was written to generate the code for evaluating these equations. This code was then included in a second program to solve the equations. Again the details of these programs are given in [3].

3.2.1. An outline of the algorithm. With the equations from the nonperiodic autocorrelation function we notice that, provided $n>6$, each equation, apart from the first, introduces four new variables. We use this to do what is effectively a tree search.

Essentially, the program works recursively by trying one set of solutions for the first four variables, then moves on to trying a set of solutions for the second group of four variables and so on, until values are assigned to all the variables. This uses about $\frac{n}{2}$ of the equations. We then use the remaining equations to confirm that these variables do form Turyn sequences.

We notice that the first two equations have respectively three and five variables. We treat them similarly by explicitly programming in trying the various sets of solutions for the three or five variables as is appropriate.

Basically, if there are four new variables that are \pm 1 , there are $2^{4}=16$ possible solutions. We could try each of these, but to minimize the searching time, we want to prune the search tree as early as possible. The best way we have found to do this is to evaluate the rest of the equation. If there is to be a solution for the four new variables, there are only five values that the rest of the equation can have. If the remainder of the equation does not equal one of these, we know we are on a wrong branch of the search tree. However, if we do get one of these values, we can cut the possible solutions down to, on average, about four sets of values and only try these solutions for the new variables. This is effectively cutting down the branching factor from sixteen to about four. Essentially, this allows us to search roughly twice as deep as with the brute-force approach.

Using this method, we can search for sequences up to about length 40 in around a CPU day on a Pyramid. Unfortunately, the increase in time is still exponential. Space requirements increase a small amount with each increase in $n$. Obviously, we need storage for about four more variables and the program will be slightly longer because of extra included code for evaluating the equations.

3.2.2. Results. We found Turyn sequences with lengths $t=2,3, \ldots, 8,13$, and 15. We found no Turyn sequences with lengths other than those just mentioned up to size $t=n+1=42$. This confirms the results of Koukouvinos, Kounias, and Sotirakoglou [12] who established the same results by a different algorithm, for $t=n+1$ up to 30 . Table 1 summarizes the number of Turyn sequences that we found. Table 2 (see p. 360) lists some of the Turyn sequences that were found. It gives one Turyn sequence of each length except for $t=13$, where there are two sequences given. From these sequences, all possible Turyn sequences of these lengths may be generated by reversing the sign of the $b_{i}$ elements and by swapping the $c$ - and $d$-sequences. 
TABLE 1. Number of Turyn sequences found

\begin{tabular}{|r|c|c|c|}
\hline$n$ & $\begin{array}{c}\text { length } \\
t\end{array}$ & $\begin{array}{c}\text { total number of } \\
\text { Turyn sequences }\end{array}$ & $\begin{array}{c}\text { decomposition } \\
(4 n+2)\end{array}$ \\
\hline 1 & 2 & 1 & $6=1^{2}+1^{2}+2^{2}+0^{2}$ \\
2 & 3 & 2 & $10=3^{1}+1^{2}+0^{2}+0^{2}$ \\
3 & 4 & 4 & $14=1^{2}+3^{2}+2^{2}+0^{2}$ \\
4 & 5 & 4 & $18=3^{2}+3^{2}+0^{2}+0^{2}$ \\
5 & 6 & 2 & $22=3^{2}+3^{2}+2^{2}+0^{2}$ \\
6 & 7 & 6 & $26=1^{2}+5^{2}+0^{2}+0^{2}$ \\
7 & 8 & 2 & $30=1^{2}+5^{2}+2^{2}+0^{2}$ \\
12 & 13 & 8 & $50=7^{2}+1^{2}+0^{2}+0^{2}$ \\
14 & 15 & 4 & $58=7^{2}+3^{2}+0^{2}+0^{2}$ \\
\hline
\end{tabular}

These transformations produce the Turyn sequences listed by Turyn in [18] and by Robinson and Seberry in [5, p. 142].

The first few cases up to $n=6$ were done by hand because the programs only deal with $n>6$. Other than the sequences listed, no Turyn sequences have been found up to $t=n+1=42$. 
TABLE 2. Turyn sequences

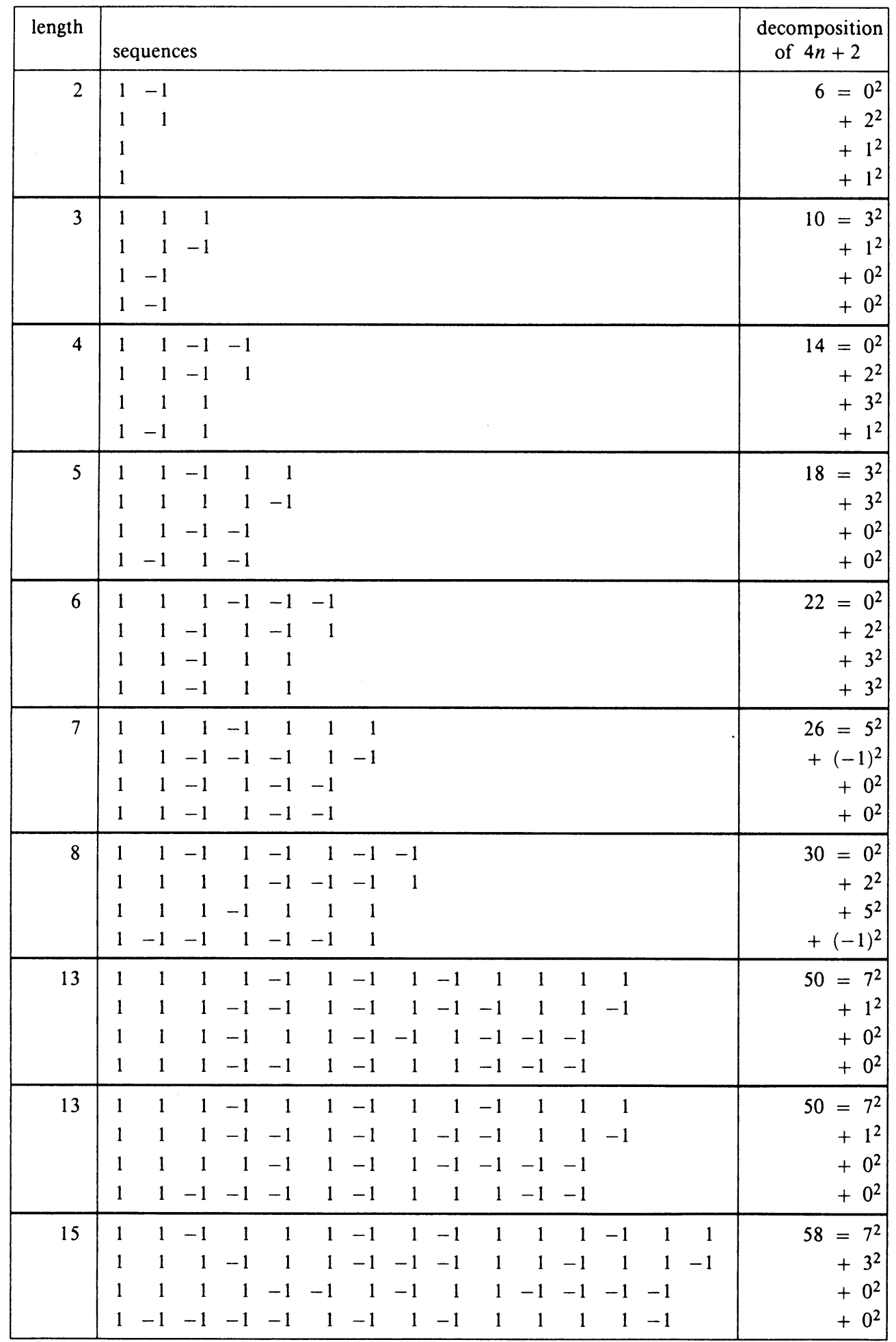


For more details about groups of four sequences with zero autocorrelation functions, we refer the interested reader to a survey by Seberry and Yamada [15] and many papers by Koukouvinos, Kounias, Seberry, Sotirakoglou [10, 11, 12, $13]$, Yang [24, 25, 26, 27], and others, which study other interesting sequences.

\section{ACKNOWLEDGMENT}

We wish to thank the referee for many valuable comments and suggestions.

\section{BIBLIOGRAPHY}

1. T. Andres, Some properties of complementary sequences, Master's Thesis, The University of Manitoba, Winnipeg, 1977.

2. H. Davenport, The higher arithmetic: An introduction to the theory of numbers, Harper Torchbooks, Harper Brothers, New York, 1960.

3. Genet M. Edmondson, More non-existent Turyn sequences, Master's Thesis, University College, University of NSW, Australian Defence Force Academy, Canberra, 1991.

4. S. Eliahou, M. Kervaire, and B. Saffari, A new restriction on the lengths of Golay complementary sequences, J. Combin. Theory Ser. A 55 (1990), 49-59.

5. A. V. Geramita and J. Seberry, Orthogonal designs: Quadratic forms and Hadamard matrices, Marcel Dekker, New York and Basel, 1979.

6. M. J. E. Golay, Complementary sequences, IRE Trans. Inform. Theory 7 (1961), 82-87.

7. _ Note on complementary series, Proc. IRE 50 (1962), 84.

8. Malcolm Griffin, There are no Golay sequences of length $2 \cdot 9^{t}$, Aequationes Math. 15 (1977), 73-77.

9. M. James, Golay sequences, Honours Thesis, University of Sydney, 1987.

10. C. Koukouvinos, S. Kounias, and J. Seberry, Further results on base sequences, disjoint complementary sequences, $O D(4 t ; t, t, t, t)$ and the excess of Hadamard matrices, Ars Combin. 30 (1990), 241-255.

11. C. Koukouvinos, C. Kounias, and K. Sotirakoglou, On Golay sequences, Discrete Math. 92 (1991), 177-185.

12. __ On base and Turyn sequences, Math. Comp. 55 (1990), 825-837.

13. C. Koukouvinos and J. Seberry, Addendum to further results on base sequences, disjoint complementary sequences, $O D(4 t ; t, t, t, t)$ and the excess of Hadamard matrices, Congr. Numer. 82 (1991), 97-103.

14. J. Kruskal, Golay's complementary series, IRE Trans. Inform. Theory 7 (1961), 273-276.

15. J. Seberry and M. Yamada, Hadamard matrices, sequences and block designs, Contemporary Design Theory-A Collection of Surveys (J. Dinitz and D. Stinson, eds.), Wiley, New York, 1992, pp. 431-560.

16. C. C. Tseng, Signal multiplexing in surface-wave delay lines using orthogonal pairs of Golay's complementary sequences, IEEE Trans. Sonics Ultrasonics 18 (1971), 103-107.

17. C. C. Tseng and C. L. Liu, Complementary sets of sequences, IEEE Trans. Inform. Theory 18 (1971), 103-107.

18. R. J. Turyn, Four-phase Barker codes, IEEE Trans. Inform. Theory 20 (1974), 366-371.

19. __ Hadamard matrices, Baumert-Hall units, four symbol sequences, pulse compression and surface wave encodings, J. Combin. Theory Ser. A 16 (1974), 313-333.

20. G. R. Welti, Quaternary codes for pulsed radar, IRE Trans. Inform. Theory Ser. A IT-6 (1960), 400-408.

21. Earl Glen Whitehead, Jr., Autocorrelation of $(+1,-1)$ sequences, Combinatorial Mathematics: Proc. Internat. Conf. (D. A. Holten and Jennifer Seberry, eds.), Lecture Notes in Math., vol. 686, Springer-Verlag, Berlin-Heidelberg-New York, 1978. 
22. C. H. Yang, Maximal binary matrices and sum of two squares, Math. Comp. 30 (1976), 148-153.

23. __ Hadamard matrices, finite sequences, and polynomials defined on the unit circle, Math. Comp. 33 (1979), 688-693.

24. __ Hadamard matrices and $\delta$-codes of length $3 n$, Proc. Amer. Math. Soc. 85 (1982), 480-482.

25. _ _ A composition theorem for $\delta$-codes, Proc. Amer. Math. Soc. 89 (1983), 375-378.

26. L L L Lagrange identities for polynomials and $\delta$-codes of lengths $7 t$ and $13 t$, Proc. Amer. Math. Soc. 88 (1983), 746-750.

27. $\ldots$, On composition of four symbol $\delta$-codes and Hadamard matrices, Proc. Amer. Math. Soc. 107 (1989), 763-776.

Department of Computer Science, University College, The University of New South Wales, Australian Defence Force Academy, Canberra ACT 2600, Australia

Department of Computer Science, The University of Wollongong, Wollongong NSW 2522, Australia

E-mail address: j.seberry@uow.edu.au

Department of Mathematics, University College, The University of New South Wales, Australian Defence Force Academy, Canberra ACT 2600, Australia 